triptolide, $6 \mu \mathrm{g}$ tridiolide or vehicle only $(1 \%$ dimethyl sulfoxide and $1 \%$ polysorbate 20 in $0.4 \mathrm{ml}$ water) for 15 weeks. Mice received treatment once daily on Monday to Friday each week.

Proteinuria and blood urea nitrogen levels were significantly reduced in both groups of treated mice compared with control mice throughout the treatment period. The survival rate of animals at age 44 weeks was significantly higher in the triptolide (87.5\%) and tripdiolide $(88.2 \%)$ groups than in the control group (35.7\%). Serum levels of antibodies to doublestranded DNA and production of interleukin-6, tumor necrosis factor and monocyte chemoattractant protein 1 by spleen mononuclear cells were all significantly lower in actively treated mice than in vehicle-treated controls.

The authors conclude that treatment with triptolide or tripdiolide reduced disease severity, increased survival and decreased cytokine production in mice with lupus nephritis. Treatment with these compounds may be beneficial in patients with lupus nephritis.

Original article Tao X et al. (2008) Effective therapy for nephritis in $(\mathrm{NZB} \times \mathrm{NZW}) \mathrm{F}_{1}$ mice with triptolide and tripdiolide, the principal active components of the Chinese herbal remedy

Tripterygium wilfordii Hook F. Arthritis Rheum 58: 1774-1783

\section{PTEN gene transfer reduces the severity of collagen-induced arthritis in rats}

Activation of the phosphatidylinositol 3 kinase/ AKT pathway is believed to promote angiogenic, proliferative and inflammatory effects in rheumatoid arthritis (RA) synovial tissue. Wang et al., therefore, tested whether negative regulation of this pathway by PTEN (phosphatidylinositol-3,4,5-trisphosphate 3-phosphatase and dual-specificity protein phosphatase) had therapeutic potential for RA. Their study investigated the effects of PTEN intra-articular gene therapy in rats with collagen-induced arthritis.

Rats' ankles were injected with adenoviral vectors encoding either human PTEN (AdPTEN) or $\beta$-galactosidase (controls) and treatment responses were monitored until day 17 . AdPTEN-treated rats had a better clinical course than control rats. Ankle joint inflammation was significantly reduced in AdPTEN-treated rats, and whereas the ankles of control rats revealed synovial hyperplasia, cartilage erosion and inflammatory infiltrates with leukocytes, those of AdPTEN-treated rats had only mild changes with no cartilage erosion. The PTEN transfer had both antiproliferative and antiangiogenic effects, since apoptosis was increased and microvessel density was decreased in the synovial tissue. Amelioration of arthritis in the ankle joints of the AdPTEN-treated mice reflected reduced levels of vascular endothelial growth factor, interleukin- $1 \beta$ and matrix metallopeptidase 9 (a protein that contributes to cartilage destruction). Furthermore, AdPTEN transfer downregulated AKT activation and reduced vascular endothelial growth factor production in human RA synovial fibroblast cells in vitro and in the ankle joints of rats with collagen-induced arthritis.

The findings hint at the potential of intra-articular gene therapy with PTEN for the treatment of RA and suggest that the phosphatidylinositol 3 kinase/AKT pathway is an important therapeutic target for the disease.

Original article Wang C-R et al. (2008) Amelioration of collagen-induced arthritis in rats by adenovirus-mediated PTEN gene transfer. Arthritis Rheum 58: 1650-1656

\section{Interferon-a2a therapy in adolescents with Behçet's disease and CNS involvement}

Central nervous system (CNS) involvement is present in $5-15 \%$ of children with Behçet's disease. Many such children do not respond to immunosuppressants, but alternative treatment with cytotoxic agents could cause serious infections or malignancy. Recombinant human interferon- $\alpha 2 a$ (rhIFN- $\alpha 2 a)$ is a treatment option in adults, but results in children have not been published. Kuemmerle-Deschner et al. now report their results with rhIFN- $\alpha 2 a$ in two boys with Behçet's disease.

The first patient, initially diagnosed with Behçet's disease at 6 years old, presented at age 14 years with neurological manifestations. MRI showed he had new brain lesions. Standard treatment did not improve his symptoms, and rhIFN- $\alpha 2$ a was initiated at $3 \times 10^{6} \mathrm{IU}$ per day, and reduced to $3 \times 10^{6} \mathrm{IU}$ three times per week after 1 month. The patient had complete disease remission after 1 month, with no disease activity at 2 years, and his CNS lesions improved. His rhIFN- $\alpha 2$ a dosage was reduced and steroids were tapered.

The second patient was diagnosed with Behçet's disease at 14 years old. His disease 